\title{
Configurational entropy of a set of dipoles placed on a two-dimensional lattice
}

\author{
P.L. Dammig Quiña, I.M. Irurzun*, E.E. Mola ${ }^{1}$ \\ CCT La Plata - CONICET, Instituto de Investigaciones Fisicoquímicas Teóricas y Aplicadas (INIFTA), Facultad de Ciencias Exactas, \\ Universidad Nacional de La Plata, Diagonal 113 y 64, CP (1900), La Plata, Argentina
}

\section{H I G H L I G H T S}

- A new expression for the entropy of adsorbed dipoles improves current predictions.

- The entropy of long molecules shows a non-monotonic dependence on the coverage.

- The calculation of the entropy of diatomic molecules for any coverage is improved.

- New formula to calculate the entropy of adsorbed dipoles as a function of coverage.

\section{A R T I C L E I N F O}

\section{Article history:}

Received 20 May 2016

Received in revised form 5 September 2016

Available online 24 September 2016

\section{Keywords:}

Entropy

Monte Carlo

Adsorption

Surfaces

Long molecules

Dipoles

\begin{abstract}
A B S T R A C T
In the present work we calculate the configurational entropy of an arbitrary number of dipoles placed on a square lattice. We use a quasi-two-dimensional (Q2D) space to capture the main features determining the occupation statistics of this system. We show that our result is in agreement with both, lattice-gas predictions at low coverages and the exact value derived in the close-packed limit as well.

Therefore our equation provides a substantial improvement to the most recent calculations based on semiempirical models and Monte Carlo simulations.
\end{abstract}

(c) 2016 Elsevier B.V. All rights reserved.

\section{Introduction}

Accurate analytical or computational calculations of the configurational entropy and free energy are a major issue to understand the thermodynamics of adsorbates.

Although the adsorption of polyatomic species was addressed long ago, the correct density dependence of the configurational entropy of a simple system such as noninteracting dimers on a two-dimensional regular lattice is still unknown. The only exact results known at present are those published by Kasteleyn [1] and Temperley et al. [2], which are valid in the close packed limit.

They found that the configurational degeneracy per lattice site was $1.791622812 \ldots$ for dimers. This value should be multiplied by $\sqrt{2}$ in the case of dipoles $(2.533737279 . .$.$) .$

Approximate methods based on the lattice-gas model that are valid at very low coverages (GD approximation) were developed [3-5]. More recently, a new EA theory to describe the adsorption of rigid rods was introduced. A semiempirical

\footnotetext{
* Corresponding author. Fax: +54 2214254642 .

E-mail address: irurzun@inifta.unlp.edu.ar (I.M. Irurzun).

1 In memory.
} 

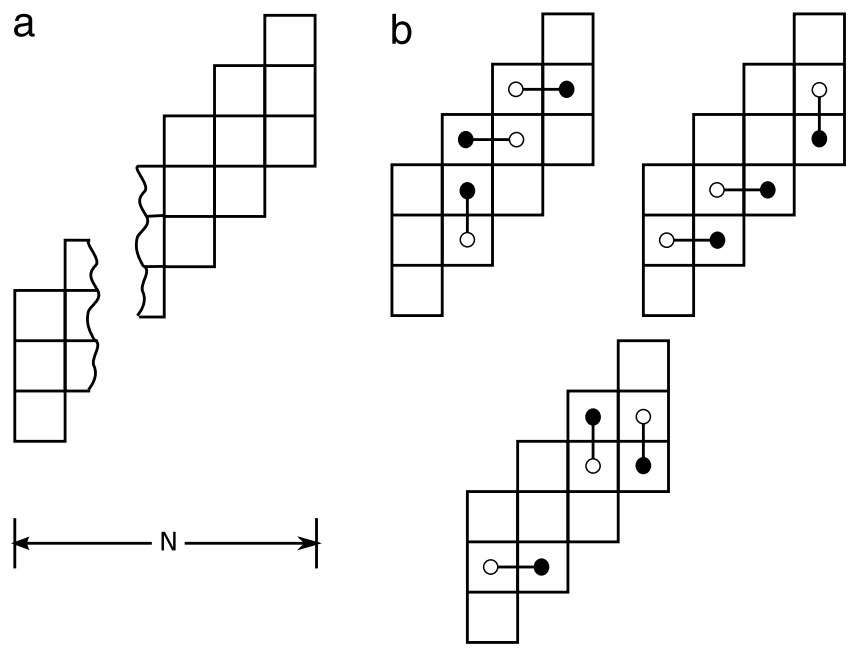

Fig. 1. (a) A $3 \times N$ diagonal array; (b) Three out of the 268 possible arrangements of three dipoles in a $3 \times 4$ Q2D space.

Table 1

Configurational degeneracy $W(k, N)$ when indistinguishable dipoles are placed in a $3 \times N$ Q2D space for $N$ and $k$ in the range $0-9$.

\begin{tabular}{|c|c|c|c|c|c|c|c|c|c|c|}
\hline \multirow[t]{2}{*}{$\mathbf{k}$} & \multicolumn{10}{|c|}{$\mathbf{N}$} \\
\hline & 0 & 1 & 2 & 3 & 4 & 5 & 6 & 7 & 8 & 9 \\
\hline 0 & $\frac{1}{2}$ & 1 & 1 & 1 & 1 & 1 & 1 & 1 & 1 & 1 \\
\hline 1 & & 4 & 12 & 20 & 28 & 36 & 44 & 52 & 60 & 68 \\
\hline 2 & & & 28 & 116 & 268 & 484 & 764 & 1,108 & 1,516 & 1,988 \\
\hline 3 & & & & 192 & 1024 & 3008 & 6,656 & 12,480 & 20,992 & 32,704 \\
\hline 4 & & & & & 1312 & 8576 & 30,496 & 79,872 & 173,600 & 332,672 \\
\hline 5 & & & & & & 8960 & 69,376 & 289,280 & 875,008 & $2,163,968$ \\
\hline 6 & & & & & & & 61,184 & 547,584 & $2,617,856$ & $8,978,944$ \\
\hline 7 & & & & & & & & 417,792 & $4,243,456$ & $22,872,064$ \\
\hline 8 & & & & & & & & & $2,852,864$ & $32,419,840$ \\
\hline 9 & & & & & & & & & & $19,480,576$ \\
\hline
\end{tabular}

(SE) model which is a combination of exact calculations in one dimension and the GD approximation at two dimensions was developed [6-9,5].

In the present work, we calculate the configurational entropy of an arbitrary number of dipoles placed on a square lattice. We show that our result is in accord with lattice-gas models and Monte Carlo simulations at very low coverages. But, even more important, it has an uncertainty of $3 \%$ with respect to the exact result in the close-packed limit. This fact represents a substantial improvement with respect to the above-mentioned developments [6,5,7-9,3].

\section{Results}

The purpose of the present paper is to provide a solution to the configurational entropy when a two-dimensional lattice space is covered by dipoles.

We use a quasi-two-dimensional (Q2D) space made up of three contiguous diagonals $3 \times N$, as is shown in Fig. 1(a), which gives the central sites of the lattice their full coordination number of nearest neighbor compartments. Fig. 1(b) shows three out of the 268 possible arrangements of two dipoles in a $3 \times 4$ Q2D space.

In a previous article [10] we used this space to exactly evaluate the configurational degeneracy $W(k, N)$ of an arbitrary number $(k)$ of dipoles placed on it. We found that $W(k, N)$ was exactly described by

$$
W(k, N)=8 W(k-1, N-1)-8 W(k-2, N-2)+W(k, N-1) .
$$

Table 1 lists the $W(k, N)$ values as a function of $k$ and $N$ in the range $0-9$. We note that in Ref. [10] the following initial conditions were stated: $W(0,1)=1, W(1,1)=4, W(0,2)=1, W(1,2)=12$ and $W(2,2)=28$. From these initial conditions we can derive $W(0,0)=\frac{1}{2}$. It should also be noticed that $W(k, N)=0$ if $k<0$ or $k>N$.

To find the generating function of the diagonal elements of Table 1, we set $N=k$ in Eq. (1) and noting that $W(k, N)=0$ if $k<0$ or $k>N$, we write

$$
W(k, k)=8 W(k-1, k-1)-8 W(k-2, k-2) .
$$


Using Zeitlin's results, Ref. [11], we can find the generating function of Eq. (2) in the following way:

$$
\frac{1}{2-16 x+16 x^{2}}=\sum_{k=0}^{\infty} W(k, k) x^{k}
$$

The generating function can also be written in the following way:

$$
\frac{1}{2-16 x+16 x^{2}}=\frac{t_{1}}{1-x T_{1}}+\frac{t_{2}}{1-x T_{2}}
$$

where $t_{1}$ and $t_{2}$ are constants, and $T_{1}$ and $T_{2}$ are the roots of the equation

$$
2 x^{2}-16 x+16=0
$$

i.e., $T_{1}=4+2 \sqrt{2}$ and $T_{2}=4-2 \sqrt{2}$. The denominator on the left-hand side of Eq. (4) has roots $T_{1}^{-1}$ and $T_{2}^{-1}$.

In the limit $x \rightarrow T_{1}^{-1}$ the first term on the right-hand side of Eq. (4) is the only dominant term, whereas in the limit $x \rightarrow T_{2}^{-1}$ the second term is the dominant one.

Therefore, the constants $t_{i}(i=1$ or 2$)$ of Eq. (4) can be determined by taking the limit

$$
\lim _{x \rightarrow T_{i}^{-1}}\left[\frac{1}{2-16 x+16 x^{2}}-\frac{t_{i}}{1-x T_{i}}\right] .
$$

Using L'Hospital's rule we determine $t_{i}$

$$
t_{i}=\frac{T_{i}^{2}}{16\left(T_{i}-2\right)} \text {. }
$$

The generating function, Eq. (4), can be rewritten as:

$$
\frac{t_{1}}{1-x T_{1}}+\frac{t_{2}}{1-x T_{2}}=\sum_{k=0}^{\infty}\left(t_{1} T_{1}^{k}+t_{2} T_{2}^{k}\right) x^{k}
$$

From Eqs. (3) and (7) we see that the diagonal elements are exactly determined by Eq. (8) for any value of $k \geq 0$.

$$
W(k, k)=t_{1} T_{1}^{k}+t_{2} T_{2}^{k}
$$

where $t_{i}$ and $T_{i}$ values have been defined above, Eqs. (5) and (6). For large values of $k$ the first term on the right-hand side of Eq. (8) will be the dominant one because $T_{1}>T_{2}>1$.

Therefore,

$$
\lim _{k \rightarrow \infty} W(k, k)=\lim _{k \rightarrow \infty} t_{1} T_{1}^{k}
$$

and

$$
\lim _{k \rightarrow \infty} \frac{W(k+1, k+1)}{W(k, k)}=T_{1}=2(2+\sqrt{2}) .
$$

For large values of $k$, the ratio between consecutive terms tends to $T_{1}$. From Table 1 we learn that this limit is approached very fast.

In the close-packed limit the number of occupied sites is $2 k$. Therefore, the root of order $2 k$ of Eq. (9) is the number of ways, $L(k, k)_{\text {dipoles }}$, in which a dipole can be placed on the central diagonal of the Q2D lattice space when $k=N$.

Therefore,

$$
L(k, k)_{\text {dipoles }}=\left(t_{1} T_{1}^{k}\right)^{\frac{1}{2 k}} .
$$

And the limit for large values of $k$ is

$$
\lim _{k \rightarrow \infty} L(k, k)_{\text {dipoles }}=T_{1}^{\frac{1}{2}}=(4+2 \sqrt{2})^{\frac{1}{2}}=2.613125 \ldots
$$

because $\left|t_{1}\right|<1$.

In order to obtain the configurational degeneracy of dimers in the close-packed limit we should divide Eq. (9) by $2^{k}$. Therefore, Eq. (11) for dimers is now written as follows:

$$
L(k, k)_{\text {dimers }}=\left(\frac{t_{1} T_{1}^{k}}{2^{k}}\right)^{\frac{1}{2 k}} .
$$

And the limit for large values of $k$ is

$$
\lim _{k \rightarrow \infty} L(k, k)_{\text {dimers }}=\left(\frac{T_{1}}{2}\right)^{\frac{1}{2}}=(2+\sqrt{2})^{\frac{1}{2}}=1.847759 \ldots
$$




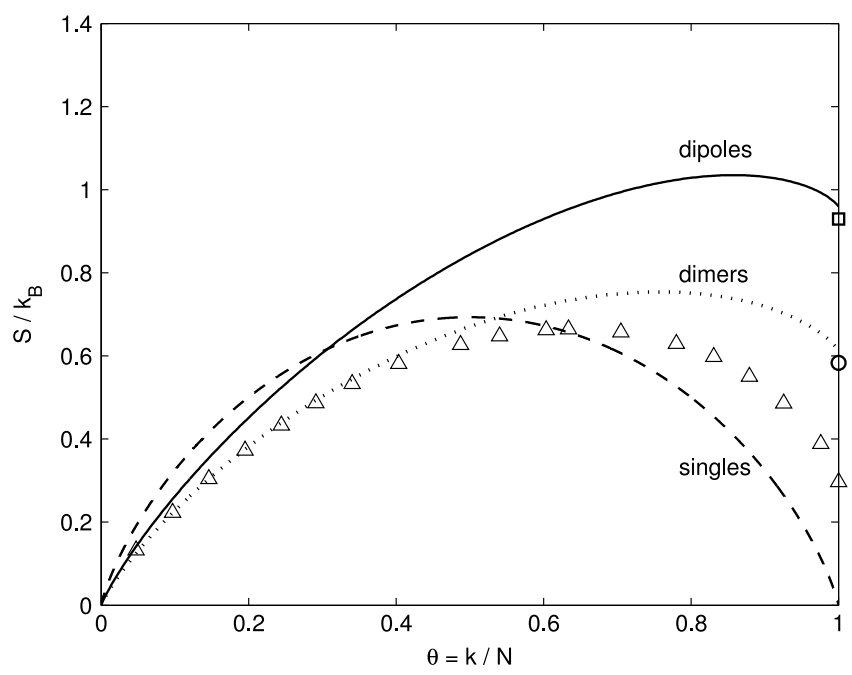

Fig. 2. Configurational entropy per lattice site as a function of coverage $\theta=\frac{k}{N}$. The circle shows the exact value derived by Kasteleyn [1] and Temperley et al. [2]. The square shows the exact value for dipoles. The triangles show Monte Carlo simulations for dimers extracted from Ref. [5].

This value is only $3.1 \%$ greater than the exact value determined in Refs. [1,2].

Following Eq. (13) we write the configurational degeneracy per lattice site, $L(k, N)$, as follows:

$$
L(k, N)=\sqrt[2 N]{W(k, N)}
$$

The root index $(2 N)$ is equal to the maximum number of lattice sites that can be occupied.

The configurational entropy per lattice site $S$ is

$$
\frac{S \text { (dipoles) }}{k_{B}}=\frac{1}{2 N} \ln W(k, N)
$$

where $k_{B}$ is the Boltzmann constant.

The curve for dimers is obtained from (16) by dividing by $2^{k}$.

Fig. 2 shows the $\frac{S}{k_{B}}$ dependence on the coverage $\theta=\frac{k}{N}$. The equation for single particles can easily be derived, and is as follows

$$
\frac{S \text { (singles) }}{k_{B}}=\ln \left[(1-\theta)^{(\theta-1)} \theta^{-\theta}\right] .
$$

To compute Eq. (16) we used a value of $N=340$ because it was the highest value our computers can manage. The difference between the value obtained by Eq. (12) at full coverage $(\theta=1)$ and the computed one for $N=340$ is less than $0.08 \%$.

In Fig. 2 the triangles show Monte Carlo simulations for dimers, based on EA theory and the SE model [5,7-9,6]. This solution agrees very well with that derived from the GD lattice-gas model for small values of coverage, as is shown and discussed in Ref. [5]. As the concentration increases, lattice-gas theories underestimate the values of $\frac{S}{k_{B}}$. Monte Carlo simulations provide a more accurate description but the disagreement is still significant for large $\theta$ 's, as can be seen in Fig. 2 by comparing with the exact result at $\theta=1$ (circle), derived by Kasteleyn [1] and Temperley et al. [2].

\section{Conclusions}

The configurational entropy of an arbitrary number of dipoles placed on a square lattice was calculated and compared with Monte Carlo results for dimmers, and with exact calculations for both dimmers (at $\theta=1$ ) and single particles in Fig. 2.

The curve for single particles starts at zero and it is symmetrical with a maximum at $\theta=0.5$ because of the indistinguishability between occupied and free sites. This symmetry is broken for long rigid molecules, and the maximum of the curve shifts to higher values of coverage because the number of possible configurations increases with the number of molecules in the lattice. In fact, this increase will contribute to the configurational entropy provided there is enough space on the lattice so that these settings can be made. Eventually, the configurational entropy decreases until $\theta=1$ because not all configurations are accessible to high coverages. It is possible to anticipate that as the molecule length increases, the configurational entropy decreases (for all $\theta>0$ ) and the maximum of the curve shifts to higher values of coverage. This general behavior is verified in simulations Monte Carlo in Refs. [5,7-9,6], though the simulations underestimate the number of possible configurations for large $\theta$ 's, leading to the mentioned disagreement in the close-packed limit and whose cause deserves to be explored.

Our equation gives a very good estimation of $\frac{S}{k_{B}}$ in the whole range of $\theta$ and therefore, it may lead to a better understanding of the adsorption properties of polyatomic species. 


\section{Acknowledgments}

This work was supported by Consejo de Investigaciones Científicas y Técnicas (CONICET), PIP 0751, Agencia Nacional de Promoción Científica y Tecnológica (ANPCyT), PICT 1036 and Universidad Nacional de La Plata, X731.

\section{References}

[1] P.W. Kasteleyn, The statistics of dimers on a lattice: I. The number of dimer arrangements on a quadratic lattice, Physica 27 (1961) $1209-1225$.

[2] H.N.V. Temperley, Michael E. Fisher, Dimer problem in statistical mechanics-an exact result, Phil. Mag. 6 (1961) 1061-1063.

[3] E.A. Guggenheim, Statistical thermodynamics of mixtures with zero energies of mixing, Proc. R. Soc. Lond. Ser. A Math. Phys. Eng. Sci. 183 (3) (1944) 203-212.

[4] E.A. DiMarzio, Statistics of orientation effects in linear polymer molecules, J. Chem. Phys. 35 (2) (1961) $658-669$.

[5] A.J. Ramirez-Pastor, P.M. Centres, Configurational entropy of adsorbed rigid rods: Theory and monte carlo simulations, Physica A 388 (10) (2009) 2001-2019

[6] D.A. Matoz-Fernandez, D.H. Linares, A.J. Ramirez-Pastor, New isotherm for multisite occupancy adsorption of long, straight rigid rods, Langmuir 27 (6) (2011) 2456-2463.

[7] A.J. Ramirez-Pastor, T.P. Eggarter, V.D. Pereyra, J.L. Riccardo, Statistical thermodynamics and transport of linear adsorbates, Phys. Rev. B 59 (1999) $11027-11036$.

[8] F. Romá, A.J. Ramirez-Pastor, J.L. Riccardo, Configurational entropy for adsorbed linear species ( $k(k$-mers)), J. Chem. Phys. 114 (2001) $10932-10937$.

[9] F. Romá, J.L. Riccardo, A.J. Ramirez-Pastor, Semiempirical model for adsorption of polyatomics, Langmuir 22 (7) (2006) $3192-3197$.

[10] P.L. Dammig Quiña, V.E. Pastor, I.M. Irurzun, E.E. Mola, Configurational degeneracy of a set of dipoles in a quasi-two-dimensional system, J. Math. Chem. 48 (2010) 592-600

[11] D. Zeitlin, On convoluted numbers and sums, Amer. Math. Monthly 74 (3) (1967) 235-246. 